\title{
Modulated infrared radiometry of rough surfaces at high temperatures
}

\author{
by B.K. BEIN (*), J.H. GU (*), A. MENSING (*), T. SOMMER (*)
}

B. WUNDERLICH (*), J. PELZL $\left({ }^{*}\right)$ and U. SEIDEL $(* *)$

(") Ruhr-Universität Bochum, Institut für Exp.-Physik, D-W-4630 Bochum, POB 102148, F.R. Germany.

(" *) Friedrich-Schiller-Universität, Inst. Optik u. Quantenelektronik, 0-0-6900 Jena, Max-Wien-Platz 1, F.R. Germany.

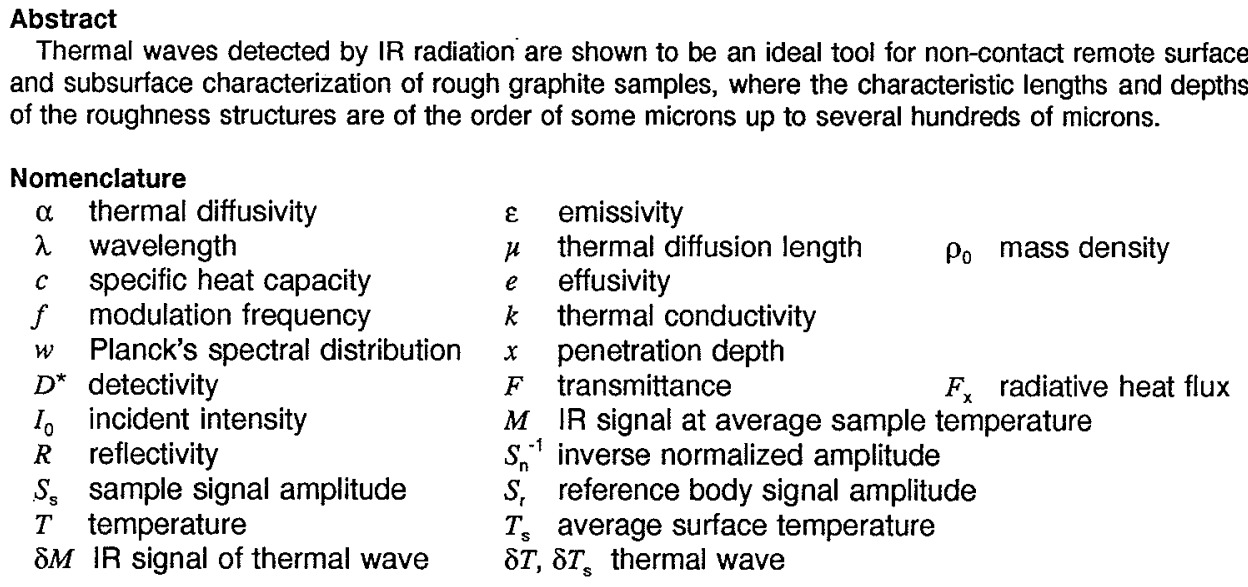

\section{Introduction}

In this work, a thermal wave system based on IR detection has been developed and its performance has been studied. The advantage of the IR radiometric technique is to be absolutely non contact and most suitable for remote sensing. It can be applied for in situ measurements of thermal properties and Non Destructive Evaluation of solid surfaces in non accessible hostile environments, e.g. at high temperatures or under irradiation by energetic particles or X-rays. In chapter 2, a short introduction to the principles of thermal depth profiling is given, followed by a description of the IR detection system. In chapter 4 thermal depth profiling studies of graphite surfaces are presented. Measurements have been done at different average sample temperatures and for various coarse roughness structures, representative for virgin and eroded graphite electrodes or graphite limiter plates. In chapter 5 the application potential of IR detection for remote measurements is analyzed.

\section{Principles of thermal wave measurements and thermal depth profiling}

Thermal waves can be excited in solids by periodically modulated heating, e.g. by a modulated laser beam. These waves are governed by the heat diffusion equation and thus are strongly damped, undergoing a phase shift with propagation. In optically opaque solids, such waves are excited just at the surface, penetrate diffusively into the solid, are reflected below the surface at local inhomogeneities of the thermal properties, and can finally be measured at the surface of the solid. To simplify the discussion of the principles of thermal wave measurements and thermal depth profiling, the example of thermal wave excitation and propagation in a homogeneous compact semi-infinite solid is discussed here. As can be seen from the solution of the heat diffusion equation: 


$$
\delta T(x, t)=\frac{(1-R)}{e} \frac{I_{0}}{\sqrt{2 \pi f}} \exp \left[-\sqrt{\frac{\pi f}{\alpha}} x\right] \cos \left[2 \pi f t-\sqrt{\frac{\pi f}{\alpha}} x-\frac{\pi}{4}\right]
$$

the amplitude has an exponential damping factor which limits the penetration depth $x$ to a distance approximately equal to the thermal diffusion length $x \propto \mu=\sqrt{\alpha / \pi f}$. The thermal diffusion length depends on the heating modulation frequency $f$, thus thermal depth profiling studies can be realized by changing the modulation frequency continuously. For high frequencies information about the effective thermal properties just at the surface is obtained, and for low modulation frequencies subsurface information from deeper below the illuminated surface is obtained. As can be seen from equation (1) the phase of thermal waves undergoes a phase shift growing with propagation distance $x$. Since amplitude damping and phase shift depend on the thermophysical parameters, measurements of the amplitude and phase give information about the effective thermal properties, both the intrinsic material parameters and topological structural properties affecting the thermal transport, such as roughness, porosity or contact resistances in layer systems. The accessible parameters are the thermal diffusivity $\alpha=k / \rho c$ and effusivity $e=\sqrt{k \rho c}$, where $k, \rho$, and $c$ are the thermal conductivity, mass density and specific heat capacity. The effusivity is the relevant thermophysical parameter for transient surface heating processes, e.g. the absorption of heat pulses, whereas the thermal diffusivity governs the heat propagation inside homogeneous solids. In general, thermal waves can also give information about optical parameters, e.g. the optical absorption constant, which describes the depth distribution of the heat sources in an illuminated solid. In equation (1) the thermal wave only depends on one optical parameter, namely the reflectivity $R$ of the opaque surface which is used to calculate the absorbed part of the incident intensity $I_{0}$.

\section{IR detection of thermal waves: Experiment and signal generation}

Thermal waves can be detected at the surface of a solid by measuring the surface temperature or the heat flux, or by measuring a temperature dependent property of the solid, e.g. the thermal expansion. In IR detection of thermal waves [1], the surface temperature of opaque solids is measured directly.

Apart from the high-temperature vacuum cell serving to keep the sample at the desired average sample temperature and protect it against oxidation and erosion, the complete measurement system (figure 1) consists of an Ar ion laser for thermal wave excitation, the IR optics and detector, the electronic components to treat the detector signal, and a computer to control the measurement and data acquisition. The radiometric signal arising from the thermal wave is a small additional oscillation of the total radiant flux, which is filtered from the high radiation level of the average sample temperature by means of a vector lock-in amplifier. This allows to analyse the thermal wave response with respect to amplitude and phase lag relative to the modulated laser beam. The reference frequency from the electro-optic modulator $C_{1}$ of the heating laser beam is used to measure the signals related to thermal waves:

$$
\delta M\left(T_{s}\right)=C \varepsilon\left(T_{s}, \lambda_{1}, \lambda_{2}\right) \int_{\lambda_{1}}^{\lambda_{2}} \mathrm{~d} \lambda F(\lambda) D^{*}(\lambda) \frac{\partial w\left(\lambda, T_{s}\right)}{\partial T_{s}} \cdot \delta T_{s}
$$

whereas the reference frequency from the mechanical chopper $\mathrm{C}_{2}$ (figure 1 ) is used to measure the radiant flux related to the average sample temperature:

$$
M\left(T_{s}\right)=C \in\left(T_{s}, \lambda_{1}, \lambda_{2}\right) \int_{\lambda_{1}}^{\lambda_{2}} \mathrm{~d} \lambda F(\lambda) D^{*}(\lambda) w\left(\lambda, T_{s}\right)
$$

In equations (2) and (3), $F(\lambda)$ is the transmittance of the IR optics, $D^{*}(\lambda)$ the detectivity of the photon detector, and $w\left(\lambda, T_{\mathrm{s}}\right)$ is Planck's spectral distribution of the blackbody radiation. The 
detectable infrared wavelength interval is limited by $\lambda_{1}<\lambda<\lambda_{2}$. The constant $C$ serves to consider the amplification factor of the detector and preamplifier, the limited solid angle of the collected radiation and the emitting surface area. As the two signals $\delta M\left(T_{\mathrm{s}}\right)$ and $M\left(T_{\mathrm{s}}\right)$ are detected with the same optical and electronic arrangement, the characteristics of the detection system and the effective emissivity of the surface $\varepsilon\left(T_{s}, \lambda_{1}, \lambda_{2}\right)$ can be eliminated, and the thermal waves:

$$
\delta T_{s}\left(T_{s}\right)=\frac{\delta M\left(T_{s}\right)}{M\left(T_{s}\right)} \cdot \frac{\int_{\lambda_{1}}^{\lambda_{2}} \mathrm{~d} \lambda F(\lambda) D^{*}(\lambda) w\left(\lambda, T_{s}\right)}{\int_{\lambda_{1}}^{\lambda_{2}} \mathrm{~d} \lambda F(\lambda) D^{*}(\lambda) \frac{\partial w\left(\lambda, T_{s}\right)}{\partial T_{s}}}
$$

can be measured directly, independently of changes of the quantity $\varepsilon\left(T_{5}, \lambda_{1}, \lambda_{2}\right)$ and long-time drifting of the experiment. A similar experimental arrangement with double modulation has been used earlier to determine both the thermal wave $\delta T_{\mathrm{s}}$ and the average sample temperature $T_{\mathrm{s}}$ [2].

The temperature rise at the surface $\delta T_{s}\left(f, T_{s}\right)$ due to thermal waves can be registered as function of the modulation frequency $f$ or alternatively as function of the average surface temperature and is interpreted with respect to amplitude and phase behaviour. If the sample is illuminated from the right hand side as shown in figure 1, reflected thermal waves are measured which give information about thermal depth profiles of surface and subsurface structures. By comparing the signals of smooth compact solids with signals measured for textured solids, one obtains information about the textures, respectively the effects of the texture, roughness or porosity on the thermal transport.

\section{Thermal depth profiles measured for graphite at different temperatures}

The aim of this work is to demonstrate the high potentiality of this method for surface and subsurface characterization of rough and porous materials. In order to avoid influences of different material properties, the surface structures were made from one graphite quality (EK 98) under equal conditions of sample preparation. The samples were cut by a saw and polished in one direction, using emery papers of different granularity $(150,800,1000,1200)$. After degassing at $250^{\circ} \mathrm{C}$ during $24 \mathrm{~h}$, the samples were free from polishing dust and adsorbed water, showing distinct roughness structures, namely macroscopic parallel grooves superimposed on the actual porosity of the graphite surfaces.

Figures 2 and 3 show the measured normalized phases of graphite samples of different roughness, measured with different detection techniques, namely photoacoustic detection [3] and IR detection. With increasing roughness, the phases in figures 2 and 3 show similar deviations from the behaviour of the homogeneous compact solid represented by the straight line. Thus we can conclude that the observed deviations are due to the structural geometrical effects on the heat transport properties and not due to pecularities of the different involved signal generation processes.

The phases of a glassy carbon sample (Sigradur K) in figure 2 are very close to the value characteristic for a compact homogeneous solid of smooth surface. For the graphite samples, however, the measured phases increase with deeper and larger grooves. However, this is only true as long as the characteristic length scale of measurement, the thermal diffusion length $\mu=\sqrt{\alpha /(\pi f)}$, is comparable with the length scales of the roughness of the sample. The phases of the rougher surface (pol. 150) are thus larger at lower frequencies and those of the less rough surfaces are larger at higher frequencies. The overall behaviour of the phases measured at 700 $\mathrm{K}$ for samples of different surface roughness (figure 3 ) confirms the results found at $300 \mathrm{~K}$. To study the effect of temperature in detail, the phases for one sample are measured at different temperatures (figure 4). The phases decrease with increasing temperature and tend to zero at higher frequencies. This means the effect of the roughness on the heat transport diminishes with increasing sample temperature where radiative contributions to the internal heat transport, $F_{x}-$ $T^{3} \cdot \operatorname{grad} T$, become more efficient. Furthermore, these radiative contributions are relatively more 
important at the shorter length scales, as can be seen in figures 3 and 4 at higher frequencies.

While the frequency-dependent phase profiles in first order give information about the thermal diffusivity and the thermal diffusion time and thus about the characteristic lengths of surface and subsurface structures, the amplitudes give information about the effusivity. Figure 5 shows the amplitude depth profiles of a well polished sample (pol. 1200) at different temperatures. The measured signals are presented as inverse normalized amplitudes, $S_{n}^{-1}=S_{r} / S_{s} \sim \sqrt{(\mathrm{kpc})_{s}}$, which are proportional to the effusivity of the sample. $S_{n}^{-1}$ is plotted versus $f^{-1 / 2}$, which means proportional to the penetration depth $x \propto \sqrt{\alpha_{s}} / \overline{\pi f}$ of the thermal wave, and thus represents the depth distribution of the effusivity. At $450 \mathrm{~K}$, a pronounced profile effect is found which can be described by a three-layer model. Just at the surface, in the limit $f^{-1 / 2} \rightarrow 0$, a very low effusivity value is measured, which can be explained by the effects of a peaked short length-scale roughness on the surface heating process [4]. In the second layer at intermediate penetration depths of the thermal wave, an increased value is found which, due to the enlarged heat capacity associated with the shadowed not directly illuminated surface areas of deep surface pores and cracks, is even higher than the effusivity of the third layer, the bulk material $[3,5]$. At low temperatures, the profile effect is more pronounced than at higher temperatures, e.g. $700 \mathrm{~K}$, where the effusivities of the three layers approach each other. The radiative contributions to the heat transport across the pores and cracks are more efficient at higher temperatures, in agreement with the phases in figure 4.

\section{Scaling of the IR detection of thermal waves}

In view of applications for non contact remote surface characterization, the scaling of the detection sensitivity with the distance between the sample (S) and the first IR lens (figure 1) has been studied as function of several experimental parmeters.

The heating modulation frequency of the light beam has been varied between $4 \mathrm{~Hz}$ and $10 \mathrm{kHz}$. Using different light sources, heating powers at the spot of $200 \mathrm{~mW}, 4 \mathrm{~W}$ and $15 \mathrm{~W}$ were realized. The emission from a surface area of about $7 \mathrm{~mm}^{2}$ has been collected for various solid angles between $0.31 \mathrm{sr}$ and $0.05 \mathrm{sr}$ and, with the help of different detectors (InAs, $\mathrm{HgCdTe}$ ), the detection wavelength intervals $1 \mu \mathrm{m}-3 \mu \mathrm{m}$ and $3.6 \mu \mathrm{m}-12 \mu \mathrm{m}$ were compared.

The lowest temperature oscillations $\delta T_{\mathrm{s}}$ detectable on sample background temperatures between $550 \mathrm{~K}$ and $750 \mathrm{~K}$ were $0.5 \mathrm{mK}-2 \mathrm{mK}$. Figure 6 shows the lowest temperature amplitudes as a function of the average sample temperature, detected for thermal waves after transmission through graphite samples of limited thickness [6]. Similar measurements were done on a Ta sample of limited thickness for the variation of the solid angle of the collected IR radiation [7]. The obtained lowest detectable temperature amplitudes were of the same order of magnitude.

Based on these results, configurations corresponding to distances of $3 \mathrm{~m}-6 \mathrm{~m}$ between the sample and the first IR lens seem to be realizable, allowing in situ measurements of thermal properties or non destructive evaluation of surfaces in non accessible hostile environments. A laser beam power of about $4.5 \mathrm{~W}$ to $15 \mathrm{~W}$ would be required.

Acknowledgement: Part of this work, the development of the IR detection system, has been supported by Max-Planck-Institut für PlasmaPhysik, Garching, FRG.

\section{REFERENCES}

[1] NORDAL (P.E.), KANSTAD (S.O.). - Infrared Physics 25, 1985, p.295-304.

[2] BUSSE (G.), EYERER (P.). - Appl.Phys.Lett. 42, 1983, p.355-357.

[3] BEIN (B.K.), KRUEGER (S.), PELZL (J.). - Can.J.Phys.64, 1986, p.1208-1216.

[4] BEIN (B.K.), WUNDERLICH (B.), PELZL (J.). - to be publ. 1993.

[5] BEIN (B.K.). - Thermal properties and photoacoustic signals of rough and porous opaque solids Statistical geometrical model considerations. In: Photoacoustic and Photothermal Phenomena (Eds. P.Hess, J.Pelzl), Springer Ser. in Opt.Sciences, Vol.58, Springer Berlin Heidelberg, 1988, p.308-311.

[6] GU (J.H.). - Doctor thesis, Ruhr-Universität Bochum, FRG, 1992.

[7] BEIN (B.K.), SOMMER (T.), SEIDEL (U.), PELZL (J.). - to be publ. 1993. 
http://dx.doi.org/10.21611/qirt.1992.062

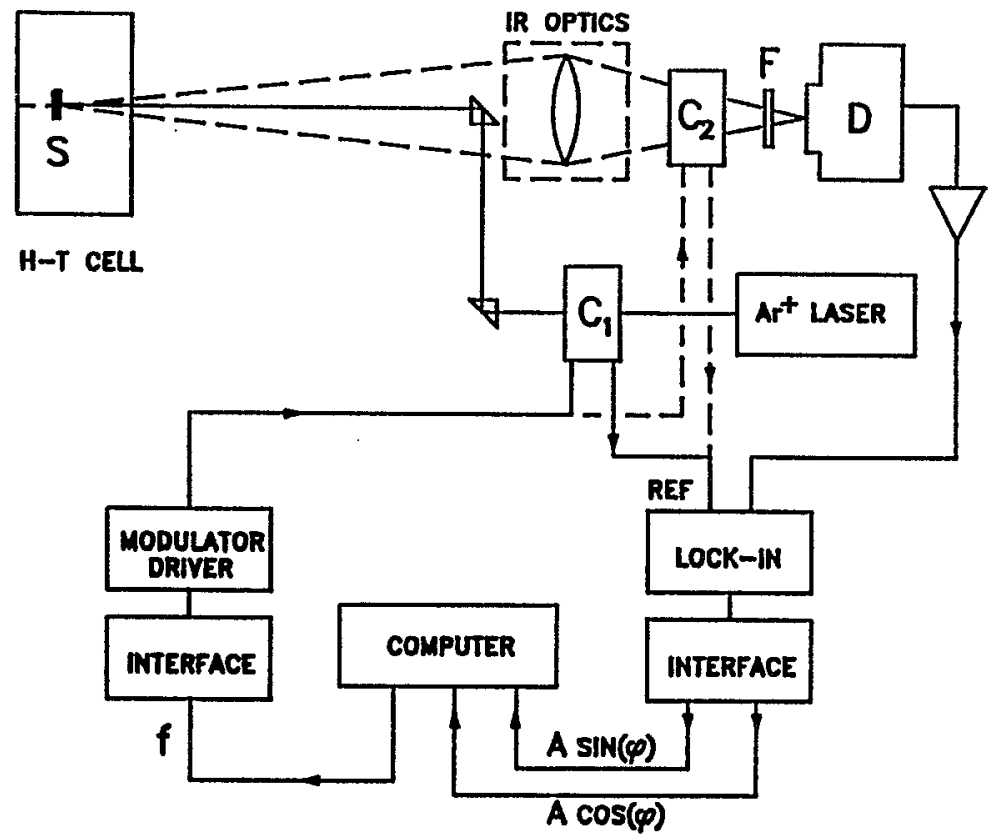

Fig. 1. - Schematic of the experimental setup, allowing to measure both the IR signal of reflected thermal waves and the radlant flux corresponding to the average surface temperature.

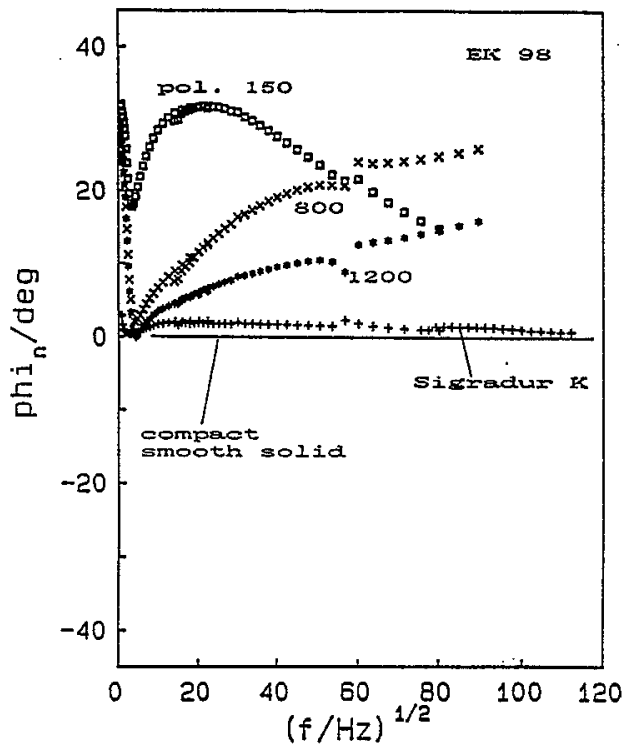

Fig.2. - Phase depth profiles of graphite samples of different surface roughness at $300 \mathrm{~K}$ (photoacoustic detection)

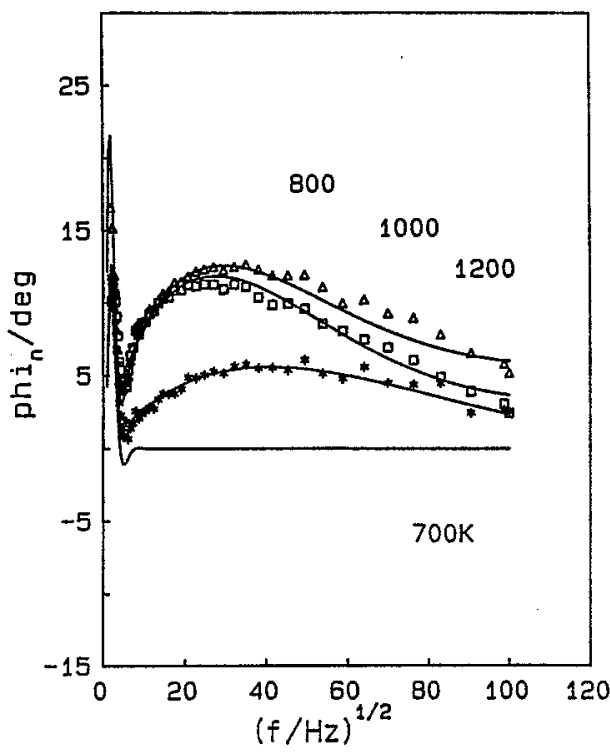

Fig. 3. - Phase depth profiles of graphite samples of different surface roughness at $700 \mathrm{~K}$ (IR detection) 
Fig.4. - Phase depth profiles of a graphite sample (pol. 800) at different temperatures .

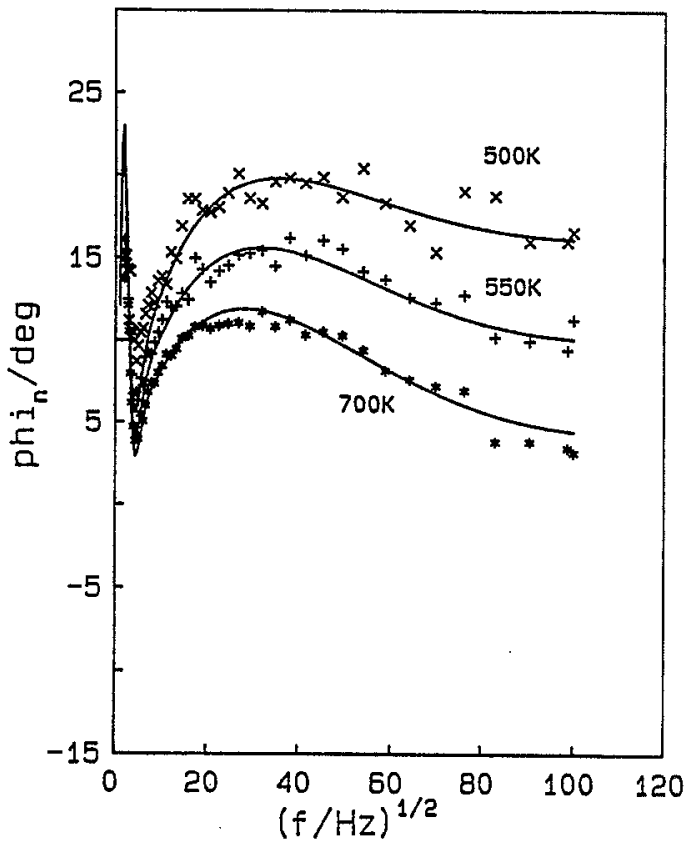

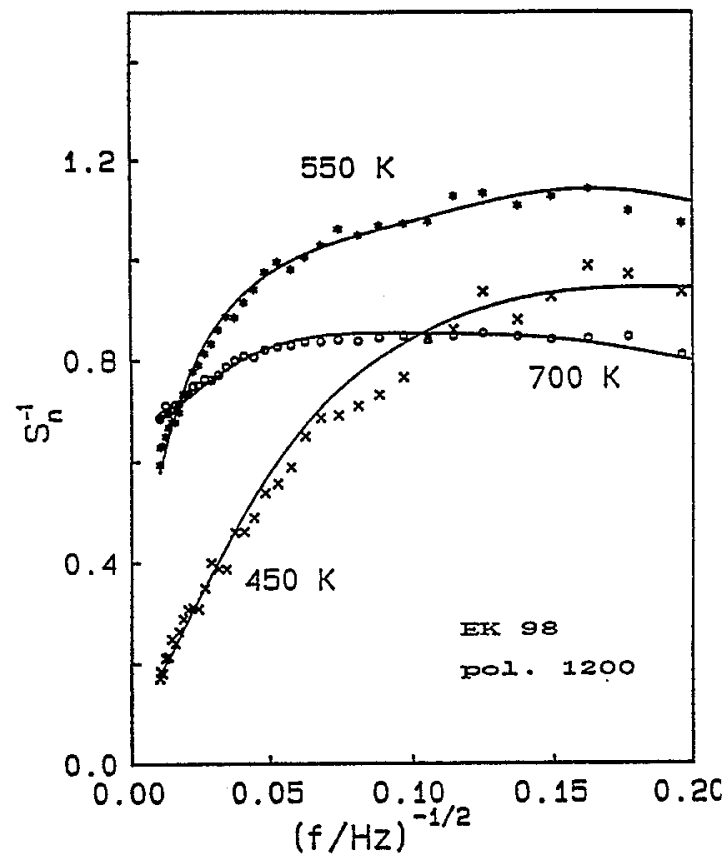

Fig.5. - Effusivity depth profiles of a well polished graphite sample (pol. 1200) at dlfferent temperatures.

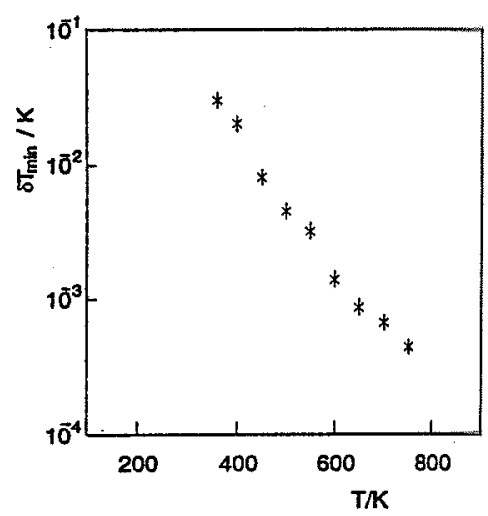

Fig. 6. - Lowest detectable temperature amplitude as function of the average sample temperature. The amplitudes were derived from transmitted thermal wave measurement on graphlte samples of limited thickness. 
http://dx.doi.org/10.21611/qirt.1992.062 